\title{
Photorealistic 3D Digital Reconstruction of a Clay Pitcher
}

\author{
Jerzy Montusiewicz', Marcin Barszcz ${ }^{1 *}$, Krzysztof Dziedzic ${ }^{1}$ \\ 1 Department of Computer Science, Faculty of Electrical Engineering and Computer Science, Lublin University \\ of Technology, 36B Nadbystrzycka, 20-618 Lublin, Poland \\ * Corresponding author's e-mail: m.barszcz@pollub.pl
}

\begin{abstract}
The availability of 3D scanning devices allows performing the process of digitisation of a growing number of diverse museum artefacts. After the properly conducted processing of the acquired point cloud, the authors obtained a photorealistic digital copy that can be presented in many ways. With regard to the objects from archaeological excavations, the vast majority are the artefacts that are more or less damaged or only partially preserved. Their state of preservation, specialised archaeological knowledge and the use of appropriate 3D computer technologies allow their complete digital reconstruction. The present work concerns the development of alternative methods and algorithms of conduct, the selection of programs and tools to recreate the surface of the outer and inner side and the bottom of a 12th century pitcher from the Afrasiab excavations in Uzbekistan.
\end{abstract}

Keywords: cultural heritage, 3D digitisation, 3D digital reconstruction, texture recreation

\section{INTRODUCTION}

The rapid development of 3D computer graphics and technologies associated with it gives great opportunities to use them in the field of museology and archeology. One of the common applications of these technologies is the creation of virtual museums (VM), which according to many authors $[1,2,3]$ are a very effective solution for communicating the cultural content due to their entertaining and educational approach. Specific examples of solutions can be found in works [4, 5,6 ], while [7] presents some guidelines regarding the development of the VR systems for cultural heritage and the methodology of creating a virtual exhibition system.

Currently, museums are interested in the digitisation of their collections not only for the preservation of cultural heritage [8], but also for the reconceptualisation of the existing exhibitions and museum functions, to create a new relationship with visitors and provide them with the content and information about the collected artefacts in a new, more attractive way [9].
The issue of employee education related to the broadly understood museology and archeology is described in [10].

The reconstruction of architectural objects can be found in [11] - a digital reconstruction of the Santa Cristina archaeological well, located near the city of Paulilatino in the Oristano province on the island of Sardinia in Italy, [12] - a three-dimensional reconstruction of the most famous archaeological site in Peru, Tschudi palace, Chan Chan.

The issue of digital reconstruction of historic buildings has already been described in several works. In [13], the procedure of a digital reconstruction of a damaged historic picture frame fragment is described by copying a similar element, and then adjusting (scaling, rotation, displacement) and placing it in the right place. Work [14] presents a digital reconstruction, and in principle the treatment of two small fragments of a large terracotta vase, found during archaeological research in the Cignan area in the south of Sicily. Specialist processing applied enabled to increase the readability of the decorations appearing on 
the elements. The authors, however, were not tempted to reconstruct the entire vase.

The author in work [15] deals with the reconstruction of the digital shape of the vase based on its elements. The characteristic points of the vase, which could be distinguished on the contour formed as a longitudinal section passing through the axis of the vase, were described. The highlighted points were EP - the vessel's limits: VT - vertical tangents: points of maximum or minimum curvature, IP - indexing points: points where the curvature's meaning changes and $\mathrm{CP}$ corner: exchange points of pronounced curvature. In addition, it analyses the position of the axis of rotation of the vessel in a situation where the vessel was made by hand and its axial symmetry is not ideal. Paper [16] presents the method of reconstructing ceramic objects having the axis of symmetry on the basis of the determined curvature signatures $\kappa(\mathrm{s})$ of the vessel fragments. This method cannot be applied because there is no set of curvature signatures for vessels and pitchers from Uzbekistan.

The aim of the work was to develop procedures allowing digital reproduction of missing areas of damaged vessels available in the form of 3D models, as well as the methods for obtaining, creating and mapping textures to cover them with generated surfaces. The prepared algorithms included the description of activities, selection of the software and tools, as well as file formats for importing and exporting objects at individual stages of operation. All activities were verified on the example of a medieval pitcher from the Afrasiab excavations in Uzbekistan.

\section{METHODOLOGY OF SURFACE RECONSTRUCTION}

\section{D scanning and postprocessing of 3D scans}

The scanning process was carried out using a structural light scanner. Scanners of this type allow for extremely fast measurements, while being characterised by high precision, even of the order of a few micrometres. They allow scanning the entire field of view, which reduces or eliminates the distortion problems caused by the scanner or object movement. Their principle of operation is that they emit a small beam of light with a specific pattern on a three-dimensional surface. This allows to precisely capture the geometric shape of the surface to the virtual world (3D object). The creation of a light beam with a specific pattern is possible owing to the built-in LCD projectors or other sources that allow the emission of stable light. Scanners are also equipped with two or more cameras, as well as the detectors that analyse and calculate the distance of each point in the field of view.

The Space Spider 3D Scanner from the Luxembourg company Artec 3D was used for the digitisation of museum objects. It is a fast 3D scanner (data acquisition speed up to 1 million points/s) with a wide practical application allowing for precise measurements in high resolution (up to $0.1 \mathrm{~mm}$ ) 'in situ'. The scanner has high 3D point accuracy of measurement (up to $0.05 \mathrm{~mm}$ ) and precise reproduction of texture and colours (texture resolution 1.3 Mpx - full colour 3D replicas). In addition, the scanner is lightweight and does not require calibration or the use of positioning markers that stick to the surface of the digitised object. For museum artefacts, it would be completely unacceptable. The scanner performs a scan using structured light. Registration of 16 frames per second allows for quick and accurate scans. Due to the low weight of the scanner $(0.85 \mathrm{~kg})$, the $3 \mathrm{D}$ digitisation process can be performed without using a tripod, from the operator's hand, and for easier manipulation of the museum artefact, it should be placed on a rotary table.

A structural light 3D scanner is a good tool for contactless and safe digitisation of museum exhibits and artefacts $[17,18]$. Its application allows developing precise 3D documentation of the analysed object and creating its faithful copy using the 3D printing technology. Owing to these possibilities, the digital preservation of museum monuments is no longer a problem. In the case of damaged exhibits, it is possible to attempt to digitally reconstruct them, so that the created exhibitions in virtual museums obtain a new dimension. They show not only in a different exhibition format, a different form, across distance over the Internet, but also the reconstructed objects, with restored original shapes, decorations and functions that they fulfilled.

\section{Methodology of surface reconstruction of 3D models of axial-symmetric objects}

When approaching the digital reconstruction of archaeological objects, it should be specified to which groups they can be ascribed, because the 
proper selection of the appropriate reconstruction method depends on it. In the case of many damaged objects or small ceramic fragments, such as pots, bowls or jugs, they can be classified as rotational objects (axially symmetrical). Archaeologists assume that their originals were made on a potter's wheel, which has been known to humanity for five thousand years. When approaching the reconstruction of this type of objects, it is very important to correctly determine their axis of symmetry and orientation in 3D space [15]. For this purpose, three basic geometrical properties found in such objects can be used: normal surface vectors of an object/fragment pass through the axis of rotation, planes perpendicular to the axis of rotation intersecting the object surface defining circles the axes of which lie on the object/fragment axis, and fragment profiles projected onto the same vertical plane occupying the same position [19].

The determination of the symmetry axis allows further work on the reconstruction of the damaged object or ceramic fragment. The 3D model can be recreated by:

- multiplication (copying) of the existing surfaces,

- modelling of the missing surfaces,

- generation of surface by rotating the profile outline,

- generation of surface by drawing a contour across a path.

\section{Texture preparation and mapping}

Digital reconstruction of the texture is a very important stage of work on the restoration of the object due to the realism of the reconstruction performed. The texture from the scanning process contains only the elements that exist on the artefact. The digitally reproduced (reconstructed) elements do not have the originally preserved texture. A solution to this problem may be the overlapping of selected areas of the original artefact texture with the reconstructed elements. The problem is the selection of the area that will ensure a photorealistic character of the entire reconstruction, adjustment of the mapped texture on the elements reconstructed to the original artefact and historical compatibility. Therefore, this process should be consulted with archaeologists and museum workers in order to ensure the greatest possible historical fidelity. In the case of maintaining small fragments of the artefact, and thus a small part of the cardboard, its digital reconstruction is an extremely complicated process that requires both the IT and history knowledge [20]. In the digital reconstruction of the texture of artefacts, one of the four basic 3D surface texture techniques can be used: UV mapping, real bitmap usage, texture painting and cloning, and bump mapping.

UV mapping involves applying a two-dimensional texture to the mesh of a three-dimensional model. The map of the 3D object grid is transformed into a plane as a 2-dimensional image; hence, a UV texture is obtained, which also has 2 dimensions with $\mathrm{U}$ and $\mathrm{V}$ coordinates. It is possible to transform the entire 3D object grid, but the effect obtained is disappointing. A more useful solution is to transform selected parts of the grid obtained by its special cutting or selected views, e.g. front, side, top.

Bitmap mapping relies on loading bitmap textures, which are usually the pictures of the surface of the real objects created, for example, in the process of 3D scanning or shooting. These textures provide the information about the original colour, glare and appearance of the artefact surface. During the reconstruction, the object is represented by a polygon grid, so the texture coordinates can be specified for each vertex of the object. In this way, the transformed mesh of the $3 \mathrm{D}$ model is linked to the $2 \mathrm{D}$ plane with the loaded bitmap texture. The effect of this is to apply and adjust the bitmap texture to the reconstructed 3D model of the museum artefact.

A useful technique for reproducing textures is also so-called textured painting and cloning. Cloning the texture involves copying a fragment from the existing texture and pasting it in the appropriate place of the 3D model grid. This allows a better fit of the texture to the 3D model.

In order to convey greater realism to the surface of the artefact, bump mapping is used. The surface of artefacts is very often not smooth and has various kinds of unevenness and roughness. Therefore, performing a mapping using a bitmap texture does not give a satisfactory effect imitating surface unevenness. The solution to this problem is the use of bump mapping technique. Such texturing simulates small surface convexity without interfering with the geometry of the 3D model. The texture used is not directly displayed, but causes local interference of the normal vector. Because each stage lighting model associates the information about the value of the angle between the ray of light and the normal vector, the result 
of the introduced disturbance is the appearance of the surface unevenness in the image. This makes the reconstructed artefact more realistic.

An important issue affecting the final result obtained is the correct implementation of the 3D model rendering process with mapped texture. Therefore, it is necessary to properly choose the types of light sources illuminating the stage, their parameters and the location of the camera.

\section{IMPLEMENTATION OF THE PROCEDURE ON THE EXAMPLE OF A PITCHER FROM AFRASIAB}

\section{Presentation of the object and its dimensional analysis}

The object under reconstruction was a broken pitcher from the turn of the 11th and 12th centuries, which was found in the Afrasiab excavation area. Afrasiab (Uzbek Afrosiyob) is an ancient city lying on the "Silk Road", inhabited since around the 5th century BC until the beginning of the 13th century when it was ravaged by the Mongol invasion. It is currently located on the northern outskirts of Samarkand and is an excavation area close to 200 ha. A pitcher with a height of approx. $35 \mathrm{~cm}$ and diameter of $21 \mathrm{~cm}$ was made of clay. The vessel has been preserved in one large fragment with the handle, the neck and the spout. The bottom and part of the side wall are missing. Hence, its digital reconstruction is difficult, because there are no unambiguous premises to determine the height of the pitcher. The pitcher does not have any decorations except for a small ribbon ornament below the neck. The simplicity of the pitcher suggests that it was intended for transporting water and served ordinary people. The pitcher from Afrasiab is currently in the Science and Practice Museum-Laboratory of the Samarkand State University (Fig. 1a). Because the object of this type was actually made using a potter's wheel, the axial symmetry of the model was checked. For this purpose, several circles were added to the external and internal walls of the model at different heights. Then, they were checked all around the perimeter to the object's walls. It turned out that the model on the section from the bottom to the neck does not have gross distortions - there are only slight deviations (Fig. 1b). The examples of values in one direction (for a selected example on the section from the base to the neck) are shown in Fig. $4 \mathrm{~b}$. The difference is $26 \mathrm{~mm}$. Then, circles were added to the neck section, the centre of which coincided with the axis of the symmetry of the model. As can be seen in Fig. 1b, the greatest deviations occur in this section (the neck does not pass perfectly through the designated axis of symmetry). However, this does not affect the reconstruction because in this part the object will not be reconstructed, since it is undamaged.

\section{D scanning and postprocessing of a pitcher}

The process of scanning a pitcher from Afrasiab was conducted 'in situ' using a Space Spider 3D Scanner by the Artec company. In the scanning process and during the processing of the data obtained, the Artec Studio version 12 Professional software was used.

This software allows the scanner to communicate with the computer and perform the scanning itself, as well as to process the data received in the scanning (point cloud) into a mesh model. In addition, it also allows applying a texture to a)

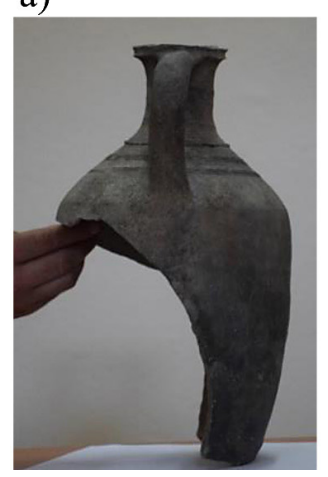

b)

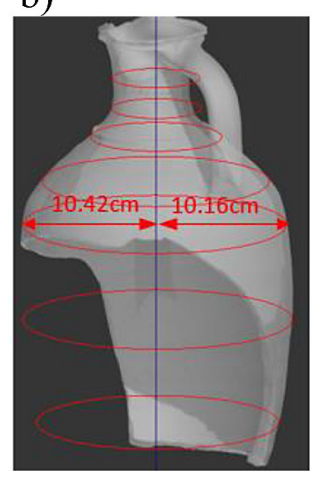

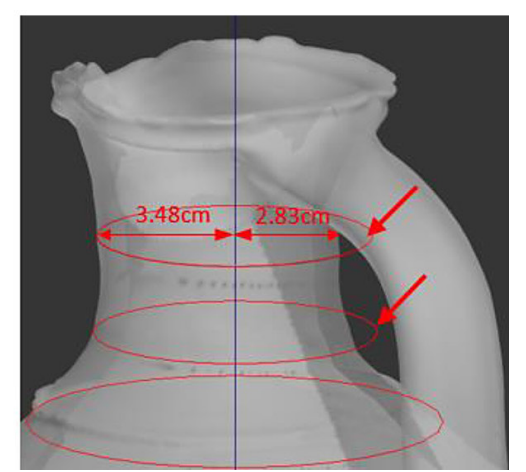

Fig. 1. Pitcher from Afrasiab: a) photo, b) matching circles to the outer surface of the pitcher on the section from the base to the neck and on the neck section 
the resulting digital model. During the digitisation of the facility, 8 scans were carried out, which enabled precise acquisition of data about the object. The data file was about 5 GB in size. Subsequent scans included the following frames: first -1728 frames, second -767 , third -783 , fourth -248 , fifth -3300 , sixth -1618 , seventh 530 , eighth -590 .The acquired cloud of points, and in addition, every few frames, the information about the object's texture was stored in each cage. A computer with an Intel Core i7 processor, 48 GB RAM and a graphics card NVIDIA Quadro K2200 with built-in 4 GB GDDR5 memory was used for the processing. The stages of the scanning and data processing process are shown in Fig. 2. The model has been exported to a file in the .obj format. This allowed storing the information about surface geometry as well as colour and texture, which is not available for the .stl format. In the case of the .obj format, the geometry is described by means of: polygons, Bezier curves or NURBS surfaces. Saving models with this extension allows for trouble-free exchange and transfer of files between graphics applications, minimising the risk of its damage.

\section{Reconstruction of the 3D model}

In order to reconstruct a damaged pitcher from Afrasiab, a 3D digital mesh model was used, which was obtained as a result of processing the data obtained from the scanning process. The reconstruction was performed in the $3 \mathrm{ds}$ Max program [21]. It allows creating and editing mesh models. After importing the 3D model in the .obj format into the program, its orientation was adjusted to the coordinate system (the pitcher axis is placed vertically), which definitely facilitated further work.

Determination of the axis of symmetry carried out according to the following procedure:

1. Determination of the approximate axis of rotation $a_{0}$ for the entire vessel,

2. Generation of $p_{i}$ planes perpendicular to the $a_{0}$ axis with a fixed pitch,

3. The determination of the $o_{i}$ circles formed by the intersection of the $p_{i}$ plane with the digital image of the vessel,

4. Designating measures for all districts of $o_{i}$,

5. Calculation of the new axis of rotation of the vessel $a_{k}$ by matching the centres of the circles by minimising the differences in position of the individual centres of $c_{i}$ in relation to the position of the axis of rotation $a_{0}$ on the plane of $p_{i}$ analysed,

$$
\begin{gathered}
a_{k}=\min \sum_{i=1}^{n} \omega_{i} \cdot \\
\cdot \sqrt{\left(a_{o_{i}}^{x}-c_{i}^{x}\right)^{2}+\left(a_{o_{i}}^{y}-c_{i}^{y}\right)^{2}}
\end{gathered}
$$

where: $\omega_{i}$ - weight of validity of calculations for each plane $p_{i}, \omega_{i}=1-$ when for plane $p_{i}$ we obtain a full circle, $\omega_{i}<1-$ for other cases, the larger the values, the longer the length of the arc created.

At the same time, these activities allowed determining the axis of symmetry (rotation) of the 3D model. For this purpose, a straight line was led across the whole 3D model through the centres of the circles. This enabled to check the deviation

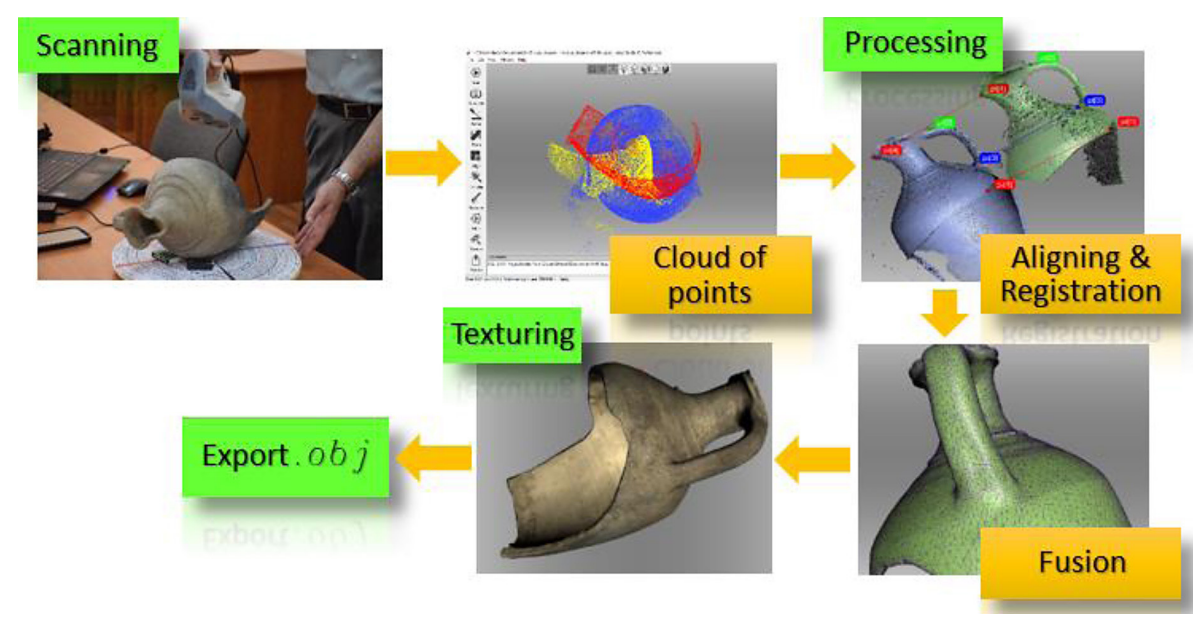

Fig. 2. Illustration of 3D scanning and data processing steps 
values of the model walls from the designated symmetry axis on the whole circumference.

At the next step, the outline was created, as shown in Fig. 3a, which was reproduced three times, and each copy was rotated with respect to the axis of symmetry by the angles of $90^{\circ}, 180^{\circ}$ and $270^{\circ}$, respectively. If the contours after rotation did not fully match the digital surfaces created after scanning, it was necessary to modify them to match the outer walls of the model (Fig. 3b). Using the Lathe tool, 3D models were made from the created outlines (Fig. 3c). In order to better match the resulting models, corresponding mesh transformations were made. Figure $3 \mathrm{c}$ shows the original model of the pitcher and its part reconstructed (the yellow line shows the outline of the original model). Both existing 3D models, after scanning and reconstruction, were combined with each other by using the Boolean algebra operation of adding.

The creation of the 3D model resulted in filling the inner space of the pitcher. In order to restore its functionality, i.e. remove the inner space of the pitcher, a copy of the reconstructed 3D model was made, and then the symmetric scaling (reduction) was selected so that the existing wall thickness was taken into account. After a proper placement of both models, the Boolean algebra operation of subtraction was used, thus removing the inner space of the pitcher, which restored its functionality. Finally, the summation of the original 3D model and the reconstructed 3D model were performed. The mesh model after the summation operation is presented in Fig. 4a. The net of the original model obtained as a result of processing data from the $3 \mathrm{D}$ scanning process is characterised by very high complexity, irregularity and is made of triangles. In the reconstructed areas, there were no points derived from the process of digitising the real object; therefore, a standard quadrangle mesh (constant mesh step) was generated, whose density of individual polygons results from the adopted program settings; hence, the $3 \mathrm{D}$ mesh is definitely simplified. The standard mesh loses its regularity in the edge area of the reconstructed model, adapting to the existing object mesh.

\section{Reconstruction of texture}

The correct mapping of textures to the surfaces of a digital model is a decisive step in obtaining photorealism of a digitally reconstructed artefact. Texturing can mask the shortcomings of the 3D geometric model to some extent.

The last stage of the digital reconstruction of the Afrasiab pitcher was the digital reconstruction of the texture. To this end, the Blender [22] program was used. The first step was to load the reconstructed 3D model and build a scene containing lighting and cameras (Fig. 4b). In order to obtain realistic scene lighting with a 3D model, the pitcher used two light sources. The Sun type light imitates the Sun rays, in which the rays run in parallel, and their intensity does not change with distance. This light generates clear strong shadows. The stage was lighted with Hemi type light, in which the rays run parallel, but do not cast shadows. They are used primarily to lighten the shadows cast by using the Sun type light.

The reconstructed digital model of the Afrasiab pitcher consists of a single mesh, which was created as a result of summing the $3 \mathrm{D}$ mesh of the original model after the scanning process and the 3D model after reconstruction. The 3D model mesh obtained from scanning is a result of triangularisation and is composed of triangles. This mesh is characterised by very high complexity. The reconstructed element of the artefact was constructed of a polygon net composed of quadrangles to obtain a simplified model (Fig. 5a). The place of summation of the grids is the area a)

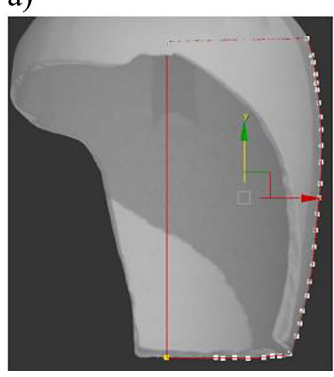

b)

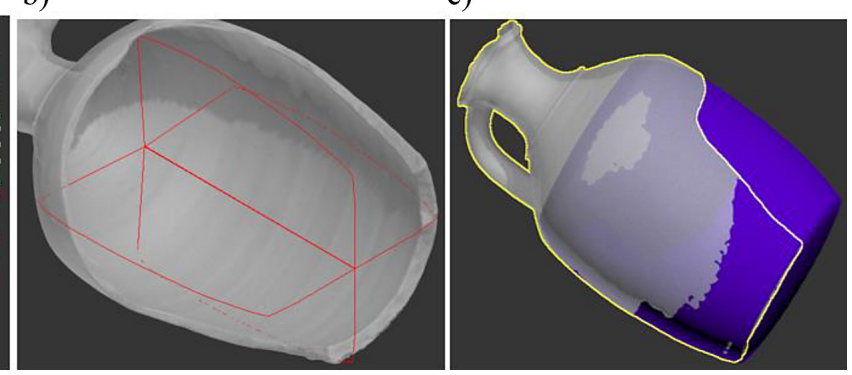

Fig. 3. The reconstruction stages: a) single contours, b) three times enlarged and rotated outlines, c) generated $3 \mathrm{D}$ model 
a)

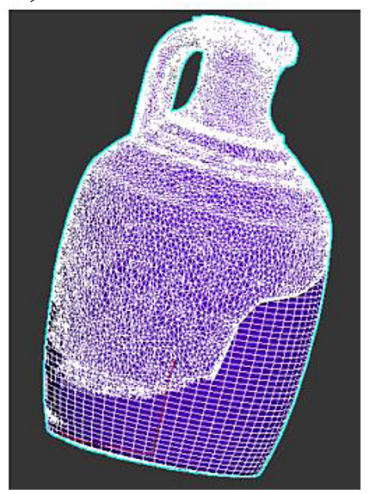

b)

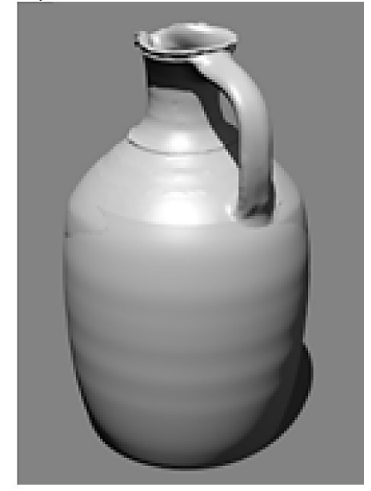

Fig. 4. A pitcher with a reconstructed part: a) in the form of a $3 \mathrm{D}$ mesh model, b) after rendering without texture

of various types of mesh deformations. These deformations are difficult to eliminate and can constitute the places of creating certain peculiarities while matching the texture of the original model with the reconstructed part. A possible but timeconsuming solution is to eliminate the duplicated vertices and vertices in which many edges coincide (Fig. 5a). The creation and application of the UV texture on the object shows the occurrence of sharp edges of the so-called "Stitches". They are most visible in the place of adding up the grids, Fig. 5b.

The next step in texturing is to create the UV layers that allow working with different 3D mesh developments on a two-dimensional plane. Bitmap textures can be loaded independently into each layer. The original texture from the 3D scanning after import to the .obj format contains a bitmap map of the images taken by the 3D scanner. Loading the original texture shows that it is only matched to the original part. However, for the reconstructed part, it is completely deformed and further extended (Fig. 5c).

The solution to this problem is UV mapping. The UV mapping of the reconstructed part consisted in transforming the selected areas of the 3D mesh into a 2-dimensional plane. This transformed mesh can undergo transformations such as scaling, rotation and offset, which allows it to be adapted to the selected bitmap texture region. Texturing in this case consisted in multiple transformations of the selected grid areas and matching them to the bitmap texture (Fig. 6a). The transformation option of Project from View was used, which transforms the selected grid in a given view. In order to compensate for sharp transitions in the joining points, texture cloning was additionally applied.

After UV mapping, the texture was saved to a .png file and then used to prepare the texture for the bump mapping process. For this purpose, , the stored texture was subjected to the process of desaturation and level correction in the Gimp program [23] (a program intended for machining two-dimensional bitmap objects), in order to sharpen the dark and light areas. Then, it was saved as a separate file. The last step was to create the material and textures for the 3D artefact model and assign to them saved bitmap textures. For a bitmap texture to the bump mapping process, it is important to set the Normal parameter, which is responsible for simulating surface unevenness. The prepared stage containing a 3D model with mapped texture, light sources and cameras was subjected to the rendering process. In order yo obtain a photorealistic effect based on the reconstructed digital model, rendering was used by means of raytracing methods. Such action allowed for a very faithful reproduction of
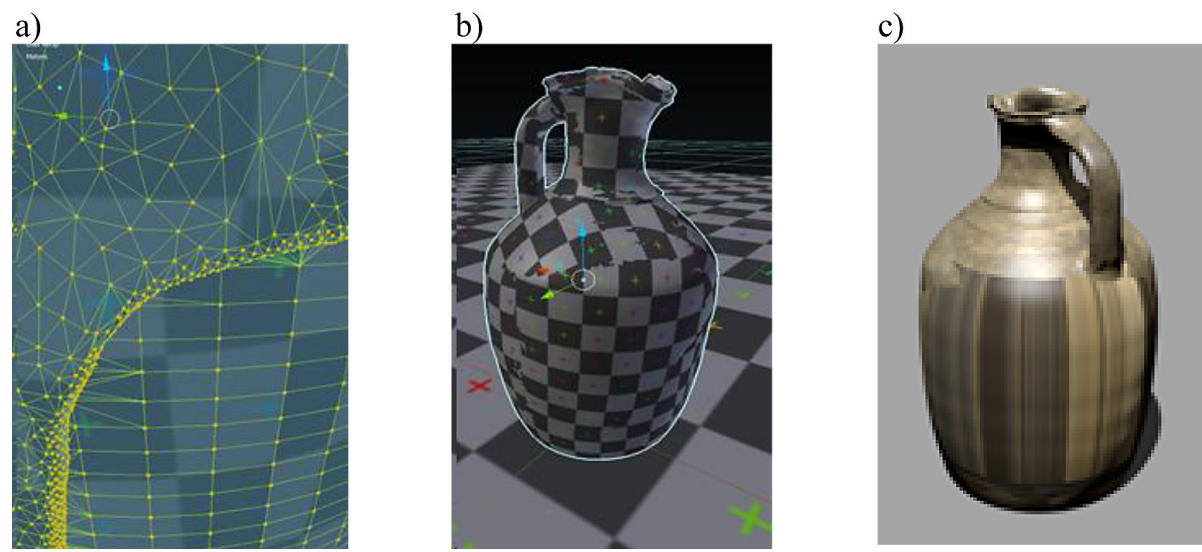

Fig. 5. Preparation for texturing: a) place of connecting the original artefact and reconstructed meshes, b) UV texture applied to the model, c) texture deformation on a reconstructed pitcher fragment 
a)

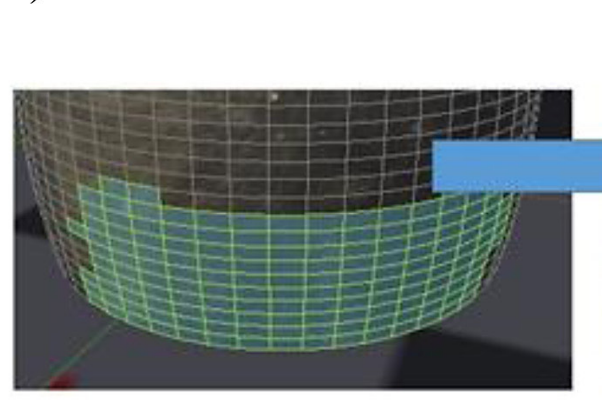

3D Model

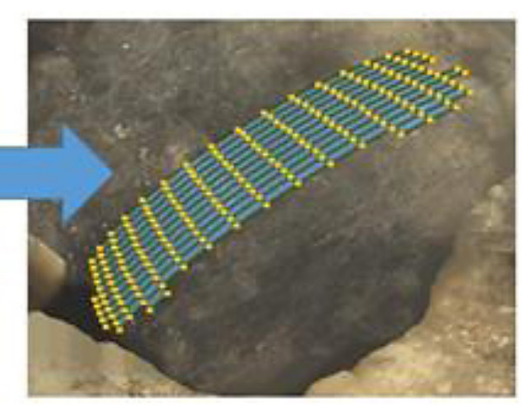

UV mapping b)

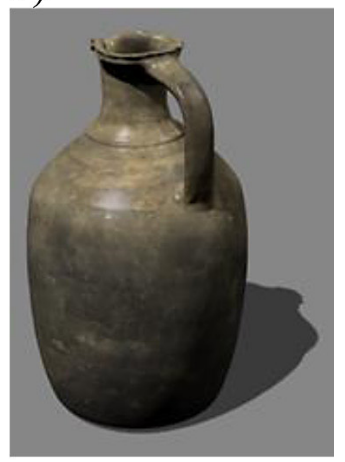

Fig. 6. Mapping: a) UV mapping made in the Blender program, b) after reconstruction via the rendering process

the texture imposed on a digital 3D model. The final effect of the digital reproduction of the pitcher from Afrasiab is visible in Figure $6 \mathrm{~b}$.

\section{CONCLUSIONS}

Preparing digital models of museum artefacts for virtual exhibitions should not be limited only to generating three-dimensional digital copies, but also to creating reconstructed objects that fully present their beauty, functions and times.

The reconstruction of the geometry and texture of museum and archaeological artefacts is usually a complex issue in terms of mathematics, IT and museology. It is an important scientific problem requiring an interdisciplinary approach, in which the people with museum and historical knowledge should be involved, apart from those familiar with modern 3D and 2D computer graphics tools, reverse engineering, graphics editing programs, and programming. Very often, it is necessary to develop a new methodology to reconstruct the object and to select the IT tools for specific cases. The digital reconstruction of the geometry and texture of the pitcher from Afrasiab enabled to formulate the following conclusions:

1. 3D scanning in structural light technology allows for effective acquisition of data under the 'in situ' conditions, which significantly simplifies the process of digitisation and the necessary formalities to be performed.

2. 3D scanning of the object and performing the appropriate postprocessing of data allows obtaining a faithful 3D mesh model with a superimposed texture that can be exported to an .obj file with a size many times smaller than the original data acquired.
3. The process of digital reconstruction of a 3D object and obtaining the effect of its photorealistic character requires the use of many specialised programs and tools. The article presents the procedure of actions that required the use of the following programs: Artec Studio version 12 Profesional, 3ds Max, Blender and Gimp.

4. The 3ds Max program proved to be useful for checking the symmetry of the pitcher and determining its axis of rotation, as well as measuring the deviations of the model's walls in relation to this axis on the entire circumference of the object. The maximum difference between the bottom and the neck is $26 \mathrm{~mm}$. Moreover, using the tools of this program, the missing part of the artefact was reconstructed and the sum of the original and reconstructed mesh was incorporated into the model of the reconstructed model grid.

5. The application of Blender and Gimp allowed for effective preparation of relevant textures and UV mapping, cloning process and bump mapping process on a digitally reconstructed 3D model of the Afrasiab pitcher.

6. Proper selection of the scene lighting and the parameters of the rendering process allowed obtaining an object with a high degree of photorealism, which confirmed the correctness of the developed procedure, applied technologies and tools for the digital reconstruction of the geometry and texture of the pitcher from Afrasiab.

\section{Acknowledgment}

The authors would like to extend their thanks to Mr Rahim Kayumov, Director of the Scientific-Experimental Museum-Laboratory of the Samarkand 
State University in Uzbekistan, for enabling us to produce multiple 3D scans of various museum artefacts and scientific consultations in the field of archaeological and museum knowledge.

\section{REFERENCES}

1. Barbieri L., Bruno F., Muzzupappa M., Virtual museum system evaluation through user studies, Journal of Cultural Heritage, 26, 2017, 101-108.

2. Bastanlar Y., Grammalidis N., Zabulis X., Yilmaz E., Yardimci Y., Triantafyllidis G., 3D reconstruction for a cultural heritage virtual tour system, ISPRS Arch. Photogramm. Remote Sens., 37(5), 2008, 1023-1028.

3. Styliani S., Fotis L., Kostas K., Petros P., Virtual museums, a survey and some issues for consideration, Journal of Cultural Heritage, 10(4), 2009, 520-528.

4. Neill S., Assessment of the NEOTHEMI virtual museum project - An on-line survey, Computers \& Education, 50(1), 2008, 410-420.

5. Robles-Ortega M.D., Feito F.R., Jiménez J.J., Segura R.J., Web technologies applied to virtual heritage: An example of an Iberian Art Museum, Journal of Cultural Heritage, 13(3), 2012, 326-331.

6. Kiourt C., Koutsoudis A., Pavlidis G., DynaMus: A fully dynamic 3D virtual museum framework, Journal of Cultural Heritage, 22, 2016, 984-991.

7. Bruno F., Bruno S., de Sensi G., Luchi M.L., Mancusoc S., Muzzupappaa M., From 3D reconstruction to virtual reality: A complete methodology for digital archaeological exhibition, Journal of Cultural Heritage, 11, 2010, 42-49.

8. Gomes L., Pereira Bellon O.R., Silva L., 3D reconstruction methods for digital preservation of cultural heritage: A survey, Pattern Recognition Letters, 50, 2014, 3-14.

9. Hashim A.F., Mohd. Taib M.Z., Alias A., The integration of interactive display method and heri-tage exhibition at museum, Procedia - Social and Behavioral Sciences, 153, 2014, 308-316.

10. Montusiewicz J., Milosz M., Kayumov R., 3D digital technologies in the practical training of archaeologists, Proceedings of the 10th International Technology, Education and Development Conference (INTED 2016), Valencia, Spain, 2016, 7451-7458.

11. Hermon S., Depalmas A., Vico Lopez M.D., Atzeni I., A 3D approach to the archaeological study of the built remains at the Santa Cristina well sanctuary,
Sardinia, Italy, Digital Applications in Archaeology and Cultural Heritage, 6, 2017, 4-9.

12. Pierdicca R., Mapping Chimu's settlements for conservation purposes using UAV and close range photogrammetry. The virtual reconstruction of Palacio Tschudi, Chan Chan, Peru, Digital Applications in Archaeology and Cultural Heritage, 8, 2018, 27-34.

13. Kęsik J., Montusiewicz J., Kayumov R., An approach to computer-aided reconstruction of museum exhibits, Advances in Science and Technology Research Journal, 11(2), 2017, 87-94.

14. Ebolese D., Lo Brutto M., Burgio A., 3d Modeling OF Two Louteria Fragments by Image-Based Approach, The International Archives of the Photogrammetry, Remote Sensing and Spatial Information Sciences, Volume XLII-5/W1, 2017, 573-580.

15. Hernandez H., 3D Reconstruction of Vessels Using 'CGVIEW', Universita' Degli Studi di Cagliari, 2012.

16. Willis A. R., Computational Analysis of Archaeological Ceramic Vessels and Their Fragments, [in] Digital Imaging for Cultural Heritage Preservation, Taylor \& Francis. 2011, 323-352.

17. Graciano A., Ortega L., Segura R.J., Feito F.R., Digitization of religious artifacts with a structured light scanner, Virtual Archaeology Review, 8(17), 2017, 49-55.

18. Adams J.W., Olah A., McCurry M.R., Potze S., Surface model and tomographic archive of fossil primate and other mammal holotype and paratype specimens of the ditsong National Museum of Natural History, Pretoria, South Africa, PLoS ONE, 10(10), 2015, 1-14.

19. Wilczek J. et al., Computer-assisted orientation and drawing of archaeological pottery, Journal on Computing and Cultural Heritage (JOCCH), 11(22), 2018, 22-38.

20. Dostala Ch., Yamafune K., Photogrammetric texture mapping: A method for increasing the Fidelity of 3D models of cultural heritage materials, Journal of Archaeological Science: Reports, 18, 2018, 430-436.

21. Derakhshani D., Autodesk 3ds Max 2016 Essentials, John Wiley \& Sons Inc, 2015.

22. Valenza E., Blender 3D Cookbook, Packt Publishing - ebooks Account, 2015.

23. Lecarme O., Delvare K., The Book of GIMP: A Complete Guide to Nearly Everything, No Starch Press, 2013. 